\title{
Entanglement conditions for multi-mode states
}

\author{
Zong-Guo Li ${ }^{1}$, Shao-Ming Fei ${ }^{1,2}$, Zhi-Xi Wang ${ }^{1}$ and $\mathrm{Ke} \mathrm{Wu}^{1}$ \\ 1 Department of Mathematics, Capital Normal University, Beijing 100037, China \\ 2 Max Planck Institute for Mathematics in the Sciences, D-04103 Leipzig, Germany
}

\begin{abstract}
We provide a class of inequalities for detecting entanglements in multi-mode systems. Necessary conditions for fully separable, bi-separable and sufficient conditions for fully entangled states are explicitly presented.
\end{abstract}

PACS numbers: 03.67.Mn

Keywords: Multi-mode state, Full entanglement 


\section{INTRODUCTION}

Entanglement plays a key role in the rapidly developing field of quantum information processing. Consequently, the study of entanglement of bi- and multipartite systems has been the focus of research in quantum information theory. In recent years, increasing attention was paid to infinite dimensional systems, the so called continuous-variable (CV) systems. In particular, Gaussian state entanglement, a special case of CV systems, has aroused great interest [1, 2, 3, 4, 5, 6, 7, 8]. The quantum teleportation network has been recently demonstrated experimentally with the use of fully symmetric three-mode Gaussian states [9]. Many efforts [2, 3, 4, 5, 7, 8, 10, 11, 12, 13, 14, 15, 16, 17] have been devoted to the study of detecting entanglement for CV systems.

It is of particular relevance to provide theoretical methods to determine the entanglement of a CV quantum system in quantum information processing and computation. Such an interest stems from the practical needs in the implementations of realistic information protocols. There has been some progress in the criteria for entanglement by uncertainty relations [10, 11, 12, 13, 14, 15]. In [13] Hillery and Zubairy have provided a class of inequalities for detecting entanglement of two-mode states.

In this paper, we generalize the inequalities to the case of multi-mode states. We derive a class of inequalities for detecting the entanglement of multi-mode states and some inequalities to detect fully entangled states. These quantities and inequalities can be, in principle, measured in standard homodyne correlation experiments [18].

\section{BIPARTITE ENTANGLED CONDITIONS FOR 3-MODE STATES}

Firstly we consider the case of three-mode states. We focus on three-mode harmonic oscillators. The derived conditions can also be applied to radiation modes, motional states of trapped ions, and related systems.

Let operators $a, b$ and $c$ be the annihilation operators of the first $(A)$, the second $(B)$ and the third $(C)$ mode, respectively. Set $L_{1}=a b c^{\dagger}+a^{\dagger} b^{\dagger} c$ and $L_{2}=i\left(a b c^{\dagger}-a^{\dagger} b^{\dagger} c\right)$. We have

$$
\left[L_{1}, L_{2}\right]=2 i\left\{N_{a} N_{b}-N_{a} N_{c}-N_{b} N_{c}-N_{c}\right\}
$$


and

$$
\begin{aligned}
\left(\Delta L_{1}\right)^{2}+\left(\Delta L_{2}\right)^{2}= & 4\left\langle N_{a} N_{b} N_{c}\right\rangle-4\left|\left\langle a b c^{\dagger}\right\rangle\right|^{2} \\
& +2\left(\left\langle N_{a} N_{b}\right\rangle+\left\langle N_{b} N_{c}\right\rangle+\left\langle N_{a} N_{c}\right\rangle+\left\langle N_{c}\right\rangle\right),
\end{aligned}
$$

where $N_{a}=a^{\dagger} a, N_{b}=b^{\dagger} b$ and $N_{c}=c^{\dagger} c$. For a pure state that is $A B \mid C$ bi-separable with respect to the first, second and third mode, we have

$$
\begin{aligned}
\left(\Delta L_{1}\right)^{2}+\left(\Delta L_{2}\right)^{2}= & 4\left\langle N_{a} N_{b}\right\rangle\left\langle N_{c}\right\rangle-4\left|\langle a b\rangle\left\langle c^{\dagger}\right\rangle\right|^{2} \\
& +2\left(\left\langle N_{a} N_{b}\right\rangle+\left\langle N_{b} N_{c}\right\rangle+\left\langle N_{a} N_{c}\right\rangle+\left\langle N_{c}\right\rangle\right) .
\end{aligned}
$$

Noting that the Schwarz inequality implies that, for any state $|\Psi\rangle,|\langle a\rangle|^{2}=|\langle\Psi|a| \Psi\rangle|^{2} \leq$ $\left|\left\langle\left.\Psi\right|^{2} \cdot|a| \Psi\right\rangle\right|^{2}=\left\langle\Psi\left|a^{\dagger} a\right| \Psi\right\rangle=\left\langle N_{a}\right\rangle,|\langle a b\rangle|^{2} \leq\left\langle N_{a} N_{b}\right\rangle$, for such a pure $A B \mid C$ separable state we get

$$
\left(\Delta L_{1}\right)^{2}+\left(\Delta L_{2}\right)^{2} \geq 2\left(\left\langle N_{a} N_{b}\right\rangle+\left\langle N_{b} N_{c}\right\rangle+\left\langle N_{a} N_{c}\right\rangle+\left\langle N_{c}\right\rangle\right)
$$

Lemma 1 For any density matrix

$$
\rho=\sum_{k} p_{k} \rho_{k}, \quad 0<p_{k} \leq 1, \quad \sum_{i} p_{i}=1
$$

and a variable $S$, we have

$$
(\Delta S)^{2} \geq \sum_{k} p_{k}\left(\Delta S_{k}\right)^{2}
$$

The lemma can be proved by the convexity. From the lemma, we have that the inequality (4) holds for $A B \mid C$ separable mixed states as well.

On the other hand the uncertainties $\Delta L_{1}$ and $\Delta L_{2}$ also satisfy

$$
\begin{aligned}
\left(\Delta L_{1}\right)^{2}+\left(\Delta L_{2}\right)^{2} & \geq 2 \Delta L_{1} \Delta L_{2} \geq\left|\left\langle\left[L_{1}, L_{2}\right]\right\rangle\right| \\
& =2\left|\left\langle N_{a} N_{c}\right\rangle-\left\langle N_{b} N_{c}\right\rangle-\left\langle N_{a} N_{c}\right\rangle-\left\langle N_{c}\right\rangle\right| .
\end{aligned}
$$

Comparing this result, which holds for any state, with inequality (44), which holds for any $A B \mid C$ separable states, we see that the right-hand side of inequality (17) is always less than or equal to that of inequality (4). Consequently, there may be states that violate inequality (4) while satisfying inequality (7). Thus we have the following theorem:

Theorem 2 If the following inequality holds for a state $\rho$,

$$
\left(\Delta L_{1}\right)^{2}+\left(\Delta L_{2}\right)^{2}<2\left(\left\langle N_{a} N_{b}\right\rangle+\left\langle N_{b} N_{c}\right\rangle+\left\langle N_{a} N_{c}\right\rangle+\left\langle N_{c}\right\rangle\right)
$$

then the state is $A B \mid C$ entangled. 
For a state $|\Psi\rangle=\left(|0\rangle_{a}|0\rangle_{b}|1\rangle_{c}+|0\rangle_{a}|1\rangle_{b}|0\rangle_{c}+|1\rangle_{a}|0\rangle_{b}|1\rangle_{c}+|1\rangle_{a}|1\rangle_{b}|0\rangle_{c}\right) / 2$, we have

$$
\left(\Delta L_{1}\right)^{2}+\left(\Delta L_{2}\right)^{2}<2\left(\left\langle N_{a} N_{c}\right\rangle+\left\langle N_{a} N_{b}\right\rangle+\left\langle N_{b} N_{c}\right\rangle+\left\langle N_{c}\right\rangle\right)
$$

Therefore the theorem shows that the state $|\Psi\rangle$ is $A B \mid C$ entangled.

Comparing (2) with (4) we have the following corollary:

Corollary 3 If the following inequality holds for a state $\rho$,

$$
\left\langle N_{a} N_{b} N_{c}\right\rangle<\left|\left\langle a b c^{\dagger}\right\rangle\right|^{2}
$$

then the state is $A B \mid C$ entangled.

Note that the Schwarz inequality implies that

$$
\left|\left\langle a b c^{\dagger}\right\rangle\right|^{2} \leq\left\langle N_{a} N_{b}\left(N_{c}+1\right)\right\rangle
$$

Formulae (8) and (9) suggest that there is a family of similar conditions for detecting entanglement, if one considers operator $a^{m} b^{n}\left(c^{\dagger}\right)^{l}$ instead of $a b c^{\dagger}$. So we have the following theorem:

Theorem 4 If the following inequality holds for a state $\rho$,

$$
\left|\left\langle a^{m} b^{n}\left(c^{\dagger}\right)^{l}\right\rangle\right|^{2}>\left\langle\left(a^{\dagger}\right)^{m} a^{m}\left(b^{\dagger}\right)^{n} b^{n}\left(c^{\dagger}\right)^{l} c^{l}\right\rangle
$$

where $m, n$ and $l$ are positive integers, then $\rho$ is $A B \mid C$ entangled.

Proof: For a $A B \mid C$ separable pure state we have

$$
\begin{aligned}
\left|\left\langle a^{m} b^{n}\left(c^{\dagger}\right)^{l}\right\rangle\right|^{2}=\left|\left\langle a^{m} b^{n}\right\rangle\right|^{2}\left|\left\langle c^{l}\right\rangle\right|^{2} & \leq\left\langle\left(a^{\dagger}\right)^{m} a^{m}\left(b^{\dagger}\right)^{n} b^{n}\right\rangle\left\langle\left(c^{\dagger}\right)^{l} c^{l}\right\rangle \\
& =\left\langle\left(a^{\dagger}\right)^{m} a^{m}\left(b^{\dagger}\right)^{n} b^{n}\left(c^{\dagger}\right)^{l} c^{l}\right\rangle .
\end{aligned}
$$

To show that formula (11) is valid for $A B \mid C$ separable mixed state (5), defining $\mathrm{A}=a^{m}$, $\mathrm{B}=b^{n}$ and $\mathrm{C}=c^{l}$, we have

$$
\begin{aligned}
\left|\left\langle\mathrm{ABC}^{\dagger}\right\rangle\right| & =\left|\sum_{k} p_{k} \operatorname{tr}\left(\rho_{k} \mathrm{ABC}^{\dagger}\right)\right| \leq \sum_{k} p_{k}\left|\operatorname{tr}\left(\rho_{k} \mathrm{ABC}^{\dagger}\right)\right| \\
& =\sum_{k} p_{k}\left|\operatorname{tr}\left(\rho_{k}^{(a b)} \mathrm{AB}\right)_{k} \operatorname{tr}\left(\rho_{k}^{(c)} \mathrm{C}^{\dagger}\right)\right| \leq \sum_{k} p_{k}\left\langle\mathrm{~A}^{\dagger} \mathrm{AB}^{\dagger} \mathrm{B}\right\rangle_{k}^{1 / 2}\left\langle\mathrm{C}^{\dagger} \mathrm{C}\right\rangle_{k}^{1 / 2}
\end{aligned}
$$


From the convexity, we have that

$$
\begin{aligned}
\left|\left\langle\mathrm{ABC}^{\dagger}\right\rangle\right| & \leq\left(\sum_{k} p_{k}\left\langle\mathrm{~A}^{\dagger} \mathrm{AB}^{\dagger} \mathrm{B}\right\rangle_{k}\left\langle\mathrm{C}^{\dagger} \mathrm{C}\right\rangle_{k}\right)^{1 / 2} \\
& =\left(\sum_{k} p_{k}\left\langle\mathrm{~A}^{\dagger} \mathrm{AB}^{\dagger} \mathrm{BC}^{\dagger} \mathrm{C}\right\rangle_{k}\right)^{1 / 2}=\left\langle\mathrm{A}^{\dagger} \mathrm{AB}^{\dagger} \mathrm{BC}^{\dagger} \mathrm{C}\right\rangle^{1 / 2}
\end{aligned}
$$

which shows that the inequality (11) does indeed hold for all $A B \mid C$ separable states. This ends the proof.

In analogy to $L_{1}$ and $L_{2}$, we have the following theorems:

Theorem 5 If any one of the following inequalities hold for a state $\rho$,

$$
\begin{gathered}
\left(\Delta J_{1}\right)^{2}+\left(\Delta J_{2}\right)^{2}<2\left(\left\langle N_{a} N_{c}\right\rangle+\left\langle N_{a} N_{b}\right\rangle+\left\langle N_{b} N_{c}\right\rangle+\left\langle N_{a}\right\rangle\right), \\
\left|\left\langle\left(a^{\dagger}\right)^{m} b^{n} c^{l}\right\rangle\right|^{2}>\left\langle\left(a^{\dagger}\right)^{m} a^{m}\left(b^{\dagger}\right)^{n} b^{n}\left(c^{\dagger}\right)^{l} c^{l}\right\rangle,
\end{gathered}
$$

where $J_{1}=a b^{\dagger} c^{\dagger}+a^{\dagger} b c, J_{2}=i\left(-a b^{\dagger} c^{\dagger}+a^{\dagger} b c\right), m, n$ and $l$ are positive integers, then the state is entangled between A-party and BC-party.

This theorem can be directly proved by using the techniques in proving theorems 2 and 4 .

We can Similarly obtain the following theorem for entangled states between B-party and AC-party:

Theorem 6 If any one of the following inequalities hold for a state $\rho$,

$$
\begin{gathered}
\left(\Delta K_{1}\right)^{2}+\left(\Delta K_{2}\right)^{2}<2\left(\left\langle N_{a} N_{c}\right\rangle+\left\langle N_{a} N_{b}\right\rangle+\left\langle N_{b} N_{c}\right\rangle+\left\langle N_{b}\right\rangle\right) . \\
\left|\left\langle a^{m}\left(b^{\dagger}\right)^{n} c^{l}\right\rangle\right|^{2}>\left\langle\left(a^{\dagger}\right)^{m} a^{m}\left(b^{\dagger}\right)^{n} b^{n}\left(c^{\dagger}\right)^{l} c^{l}\right\rangle
\end{gathered}
$$

where $K_{1}=a b^{\dagger} c+a^{\dagger} b c^{\dagger}$ and $K_{2}=i\left(a b^{\dagger} c-a^{\dagger} b c^{\dagger}\right), m, n$ and $l$ are positive integers, then the state is $B \mid A C$ entangled.

In fact, there is another class of inequalities detecting the bipartite entangled states. We have the following theorem:

Theorem 7 For a state $\rho$,

if $\left|\left\langle a^{m} b^{n} c^{l}\right\rangle\right|>\left[\left\langle\left(a^{\dagger}\right)^{m} a^{m}\right\rangle\left\langle\left(b^{\dagger}\right)^{n} b^{n}\left(c^{\dagger}\right)^{l} c^{l}\right\rangle\right]^{1 / 2}$, then the state is $A \mid B C$ entangled; if $\left|\left\langle a^{m} b^{n} c^{l}\right\rangle\right|>\left[\left\langle\left(b^{\dagger}\right)^{n} b^{n}\right\rangle\left\langle\left(a^{\dagger}\right)^{m} a^{m}\left(c^{\dagger}\right)^{l} c^{l}\right\rangle\right]^{1 / 2}$, then the state is $B \mid A C$ entangled; if $\left|\left\langle a^{m} b^{n} c^{l}\right\rangle\right|>\left[\left\langle\left(a^{\dagger}\right)^{m} a^{m}\left(b^{\dagger}\right)^{n} b^{n}\right\rangle\left\langle\left(c^{\dagger}\right)^{l} c^{l}\right\rangle\right]^{1 / 2}$, then the state is $A B \mid C$ entangled . 
Proof: We prove the condition for $A \mid B C$ entanglement.

From Schwarz inequality, we have

$$
\left|\left\langle a^{m} b^{n} c^{l}\right\rangle\right| \leq\left[\left\langle\left(a^{\dagger}\right)^{m} a^{m}\right\rangle\left\langle\left(b^{\dagger}\right)^{n} b^{n}\left(c^{\dagger}\right)^{l} c^{l}\right\rangle\right]^{1 / 2}
$$

for a pure $A \mid B C$ separable state.

For $A \mid B C$ separable mixed state $\rho=\sum_{k} p_{k} \rho_{k}$, where $\rho_{k}$ is the density matrix corresponding to a pure $A \mid B C$ separable state, and $p_{k}$ is the probability of $\rho_{k}$, we have

$$
\begin{aligned}
|\langle\mathrm{ABC}\rangle|^{2} & =\left|\sum_{k} p_{k} \operatorname{tr}\left(\rho_{k} \mathrm{ABC}\right)\right|^{2} \leq\left(\sum_{k} p_{k}\left|\operatorname{tr}\left(\rho_{k} \mathrm{ABC}\right)\right|\right)^{2} \\
& =\sum_{i, j} p_{i} p_{j}\left|\langle\mathrm{~A}\rangle_{i}\langle\mathrm{BC}\rangle_{i}\right|\left|\left\langle\mathrm{C}^{\dagger} \mathrm{B}^{\dagger}\right\rangle_{j}\left\langle\mathrm{~A}^{\dagger}\right\rangle_{j}\right| \\
& \leq \sum_{i, j} p_{i} p_{j}\left(\left\langle\mathrm{~A}^{\dagger} \mathrm{A}\right\rangle_{i}\left\langle\mathrm{~B}^{\dagger} \mathrm{BC}^{\dagger} \mathrm{C}\right\rangle_{i}\left\langle\mathrm{~B}^{\dagger} \mathrm{BC}^{\dagger} \mathrm{C}\right\rangle_{j}\left\langle\mathrm{~A}^{\dagger} \mathrm{A}\right\rangle_{j}\right)^{1 / 2}
\end{aligned}
$$

where $\mathrm{A}=a^{m}, \mathrm{~B}=b^{n}$ and $\mathrm{C}=c^{l}$. Set $\left\langle\mathrm{A}^{\dagger} \mathrm{A}\right\rangle_{i}=x_{i},\left\langle\mathrm{~B}^{\dagger} \mathrm{BC}^{\dagger} \mathrm{C}\right\rangle_{i}=y_{i}$. (19) can be expressed as

$$
|\langle\mathrm{ABC}\rangle|^{2} \leq \sum_{k} p_{k}^{2} x_{k} y_{k}+2 \sum_{i<j} p_{i} p_{j}\left(x_{i} y_{i} x_{j} y_{j}\right)^{1 / 2}
$$

While $\left\langle\mathrm{A}^{\dagger} \mathrm{A}\right\rangle\left\langle\mathrm{B}^{\dagger} \mathrm{BC}^{\dagger} \mathrm{C}\right\rangle$ can be expressed as

$$
\left\langle\mathrm{A}^{\dagger} \mathrm{A}\right\rangle\left\langle\mathrm{B}^{\dagger} \mathrm{BC}{ }^{\dagger} \mathrm{C}\right\rangle=\sum_{k} p_{k}^{2} x_{k} y_{k}+\sum_{i<j} p_{i} p_{j}\left(x_{i} y_{j}+x_{j} y_{i}\right)
$$

Noting that $x_{i} y_{j}+x_{j} y_{i} \geq 2\left(x_{i} y_{i} x_{j} y_{j}\right)^{1 / 2}$, we see that from (20) and (21) the inequality (18) holds for all $A \mid B C$ bipartite separable states. Hence if a state violates this inequality (18), it must be bipartite entangled between A-part and BC-part. The other conditions can be proved similarly.

For $m=n=l=1$, formula (18) implies that for a $A \mid B C$ separable state

$$
|\langle a b c\rangle|^{2} \leq\left\langle N_{a}\right\rangle\left\langle N_{b} N_{c}\right\rangle
$$

For an arbitrary state, we have $|\langle a b c\rangle|^{2} \leq\left[\left\langle N_{a}+1\right\rangle\left\langle N_{b} N_{c}\right\rangle\right]$. Therefore there may be states that do violate the inequality (22) and are $A \mid B C$ entangled at the same time.

\section{FULLY ENTANGLED CONDITIONS FOR 3-MODE STATES}

We first introduce the notions of fully separable 3-mode states and fully entangled ones in[19, 20]. A 3-mode state is fully separable if and only if the state can be described as 
mixtures of $\rho_{1} \otimes \rho_{2} \otimes \rho_{3}$. A 3-mode entangled state is fully entangled if it is not bi-partite separable. We have our main theorem:

Theorem 8 If the following inequality holds for a state $\rho$,

$$
(\Delta K(\phi))^{2}<1
$$

where $K(\phi)=e^{i \phi} a^{\dagger} b^{\dagger} c^{\dagger}+e^{-i \phi} a b c, \phi \in[0,2 \pi]$, then the state is fully entangled.

Proof:

$$
\begin{aligned}
(\Delta K(\phi))^{2}= & e^{2 i \phi}\left\langle\left(a^{\dagger} b^{\dagger} c^{\dagger}-\left\langle a^{\dagger} b^{\dagger} c^{\dagger}\right\rangle\right)^{2}\right\rangle+e^{-2 i \phi}\left\langle(a b c-\langle a b c\rangle)^{2}\right\rangle \\
& +\left\langle\left(a^{\dagger} b^{\dagger} c^{\dagger}-\left\langle a^{\dagger} b^{\dagger} c^{\dagger}\right\rangle\right)(a b c-\langle a b c\rangle)\right\rangle \\
& +\left\langle(a b c-\langle a b c\rangle)\left(a^{\dagger} b^{\dagger} c^{\dagger}-\left\langle a^{\dagger} b^{\dagger} c^{\dagger}\right\rangle\right)\right\rangle .
\end{aligned}
$$

As $e^{2 i \phi}\left\langle\left(a^{\dagger} b^{\dagger} c^{\dagger}-\left\langle a^{\dagger} b^{\dagger} c^{\dagger}\right\rangle\right)^{2}\right\rangle+e^{-2 i \phi}\left\langle(a b c-\langle a b c\rangle)^{2}\right\rangle$ is real, by using the Schwarz inequality

$$
\begin{aligned}
& \left|\left\langle(a b c-\langle a b c\rangle)^{2}\right\rangle\right| \\
\leq & {\left[\left\langle(a b c-\langle a b c\rangle)\left(a^{\dagger} b^{\dagger} c^{\dagger}-\left\langle a^{\dagger} b^{\dagger} c^{\dagger}\right\rangle\right)\right\rangle\left\langle\left(a^{\dagger} b^{\dagger} c^{\dagger}-\left\langle a^{\dagger} b^{\dagger} c^{\dagger}\right\rangle\right)(a b c-\langle a b c\rangle)\right\rangle\right]^{1 / 2}, }
\end{aligned}
$$

we obtain

$$
\begin{aligned}
& (\Delta K(\phi))^{2} \\
\geq & -\left|\left\langle\left(a^{\dagger} b^{\dagger} c^{\dagger}-\left\langle a^{\dagger} b^{\dagger} c^{\dagger}\right\rangle\right)^{2}\right\rangle\right|-\left|\left\langle(a b c-\langle a b c\rangle)^{2}\right\rangle\right| \\
& +\left\langle\left(a^{\dagger} b^{\dagger} c^{\dagger}-\left\langle a^{\dagger} b^{\dagger} c^{\dagger}\right\rangle\right)(a b c-\langle a b c\rangle)\right\rangle+\left\langle(a b c-\langle a b c\rangle)\left(a^{\dagger} b^{\dagger} c^{\dagger}-\left\langle a^{\dagger} b^{\dagger} c^{\dagger}\right\rangle\right)\right\rangle \\
\geq & -2\left[\left\langle(a b c-\langle a b c\rangle)\left(a^{\dagger} b^{\dagger} c^{\dagger}-\left\langle a^{\dagger} b^{\dagger} c^{\dagger}\right\rangle\right)\right\rangle\left\langle\left(a^{\dagger} b^{\dagger} c^{\dagger}-\left\langle a^{\dagger} b^{\dagger} c^{\dagger}\right\rangle\right)(a b c-\langle a b c\rangle)\right\rangle\right]^{1 / 2} \\
& +\left\langle\left(a^{\dagger} b^{\dagger} c^{\dagger}-\left\langle a^{\dagger} b^{\dagger} c^{\dagger}\right\rangle\right)(a b c-\langle a b c\rangle)\right\rangle+\left\langle(a b c-\langle a b c\rangle)\left(a^{\dagger} b^{\dagger} c^{\dagger}-\left\langle a^{\dagger} b^{\dagger} c^{\dagger}\right\rangle\right)\right\rangle \\
= & {\left[\left(\left\langle\left(N_{a}+1\right)\left(N_{b}+1\right)\left(N_{c}+1\right)\right\rangle-|\langle a b c\rangle|^{2}\right)^{1 / 2}-\left(\left\langle N_{a} N_{b} N_{c}\right\rangle-|\langle a b c\rangle|^{2}\right)^{1 / 2}\right]^{2} . }
\end{aligned}
$$

The inequality (26) is valid for all states. In particular, if the state is a fully separable pure state, (26) becomes

$$
\begin{array}{r}
(\Delta K(\phi))^{2} \geq\left[\left(\left\langle N_{a}+1\right\rangle\left\langle N_{b}+1\right\rangle\left\langle N_{c}+1\right\rangle-|\langle a b c\rangle|^{2}\right)^{1 / 2}\right. \\
\left.-\left(\left\langle N_{a}\right\rangle\left\langle N_{b}\right\rangle\left\langle N_{c}\right\rangle-|\langle a b c\rangle|^{2}\right)^{1 / 2}\right]^{2} .
\end{array}
$$

Denote $x_{1}=\left\langle N_{a}\right\rangle, x_{2}=\left\langle N_{b}\right\rangle, x_{3}=\left\langle N_{c}\right\rangle$ and $z=|\langle a b c\rangle|^{2}$. Let us find the minimum of the function

$$
F\left(x_{1}, x_{2}, x_{3}\right)=\sqrt{\left(x_{1}+1\right)\left(x_{2}+1\right)\left(x_{3}+1\right)-z}-\sqrt{x_{1} x_{2} x_{3}-z},
$$


in the region $x_{1} x_{2} x_{3} \geq z \geq 0$.

There are two cases: 1. $F\left(x_{1}, x_{2}, x_{3}\right)$ has minimum in the region of interest. From $\partial F\left(x_{1}, x_{2}, x_{3}\right) / \partial x_{i}=0, i=1,2,3, F$ acquires the minimum at $x_{1}=x_{2}=x_{3}=x$,

$$
F_{\min }=\sqrt{(x+1)^{3}-z}-\sqrt{x^{3}-z} .
$$

As $\left(\sqrt{(x+1)^{3}-z}\right)^{2}-\left(1+\sqrt{x^{3}-z}\right)^{2} \geq(x+1)^{3}-\left(x^{3}+1+2 \sqrt{x^{3}}\right)$, we have $F_{\min } \geq 1$ in the region of interest.

2. $F\left(x_{1}, x_{2}, x_{3}\right)$ has no local minimum in the region of interest. Then the minimum of the function must lie on the boundary, $x_{1} x_{2} x_{3}=z$ or at least one of the $x_{1}, x_{2}$ and $x_{3}$ goes to infinity.

On the boundary $x_{1} x_{2} x_{3}=z$ we have

$$
F\left(x_{1}, x_{2}, x_{3}\right)=\sqrt{\left(x_{1}+1\right)\left(x_{2}+1\right)\left(x_{3}+1\right)-x_{1} x_{2} x_{3}} \geq 1 .
$$

When $x_{1}, x_{2}, x_{3} \longrightarrow \infty$, we obtain

$$
\begin{aligned}
F\left(x_{1}, x_{2}, x_{3}\right)= & \int_{x_{1} x_{2} x_{3}-z}^{\left(x_{1}+1\right)\left(x_{2}+1\right)\left(x_{3}+1\right)-z} \frac{1}{2 \sqrt{u}} d u \\
\geq & \frac{\left(x_{1}+1\right)\left(x_{2}+1\right)\left(x_{3}+1\right)-x_{1} x_{2} x_{3}}{2 \sqrt{\left(x_{1}+1\right)\left(x_{2}+1\right)\left(x_{3}+1\right)-z}} \\
\geq & \frac{\left(x_{1}+1\right)\left(x_{2}+1\right)\left(x_{3}+1\right)-x_{1} x_{2} x_{3}}{2 \sqrt{\left(x_{1}+1\right)\left(x_{2}+1\right)\left(x_{3}+1\right)}} \\
= & \frac{1}{2}\left[\sqrt{\frac{x_{1}+1}{\left(x_{2}+1\right)\left(x_{3}+1\right)}}+\sqrt{\frac{\left(x_{2}+1\right)\left(x_{3}+1\right)}{x_{1}+1}}\right. \\
& \left.+\frac{x_{1} x_{2}+x_{1} x_{3}-1}{\sqrt{\left(x_{1}+1\right)\left(x_{2}+1\right)\left(x_{3}+1\right)}}\right] \\
\geq & 1+\frac{x_{1} x_{2}+x_{1} x_{3}-1}{2 \sqrt{\left(x_{1}+1\right)\left(x_{2}+1\right)\left(x_{3}+1\right)}} .
\end{aligned}
$$

When one of the $x_{1}, x_{2}$ and $x_{3}$ goes to infinity, while the rest keep finite, the proof can be done similarly. Therefore, $F_{\min } \geq 1$ holds for any fully separable pure states. Finally, we have, from Lemma 1,

$$
(\Delta K(\phi))^{2} \geq 1
$$

for fully separable mixed states. 
Actually inequality (32) is also true for any bi-separable pure states. For example, for a $A \mid B C$ separable pure state, we have

$$
(\Delta K(\phi))^{2} \geq\left[\left(\left\langle N_{a}+1\right\rangle\left\langle\left(N_{b}+1\right)\left(N_{c}+1\right)\right\rangle-|\langle a b c\rangle|^{2}\right)^{1 / 2}-\left(\left\langle N_{a}\right\rangle\left\langle N_{b} N_{c}\right\rangle-|\langle a b c\rangle|^{2}\right)^{1 / 2}\right]^{2} .
$$

Setting $x_{1}=\left\langle N_{a}\right\rangle, x_{2}=\left\langle N_{b} N_{c}\right\rangle, x_{3}=\left\langle N_{b}\right\rangle, x_{4}=\left\langle N_{c}\right\rangle$ and $z=|\langle a b c\rangle|^{2}$, we define a function

$$
\begin{aligned}
G\left(x_{1}, x_{2}, x_{3}, x_{4}, z\right) & =\sqrt{\left(x_{1}+1\right)\left(x_{2}+x_{3}+x_{4}+1\right)-z}-\sqrt{x_{1} x_{2}-z} \\
& \geq \sqrt{\left(x_{1}+1\right)\left(x_{2}+1\right)-z}-\sqrt{x_{1} x_{2}-z}
\end{aligned}
$$

which is greater than or equal to 1 in the region of interest. From lemma 1, we know that (32) is also valid for any bi-partite separable mixed states. Therefore violation of inequality (32) implies full entanglement. This ends the proof.

As an example we consider the GHZ state, $|G H Z\rangle=(|000\rangle+|111\rangle) / \sqrt{2}$. For this state, we have $(\Delta K)^{2}=\left(\left\langle K^{2}\right\rangle\right)-(\langle K\rangle)^{2}=0$, where $K=K(\phi=0)$. Thus the GHZ state is fully entangled.

In analogy to (11), it is possible to find other relations satisfied by separable states. For example, in the case of pure fully separable states we have that

$$
|\langle a b c\rangle|=|\langle a\rangle\langle b\rangle\langle c\rangle| \leq\left[\left\langle N_{a}\right\rangle\left\langle N_{b}\right\rangle\left\langle N_{c}\right\rangle\right]^{1 / 2}
$$

However, (35) is not always true for some fully separable mixed states. In fact, for a tripartite pure state, we can detect a fully entangled state with respect to all three bipartite decompositions. If all three bipartite decompositions are entangled, the tripartite state is fully entangled.

Here is another criterion for detecting pure fully entangled states.

Theorem 9 If a pure state violates inequality (35) and satisfies the following relations simultaneously,

$$
\begin{aligned}
& |\langle a\rangle\langle b c\rangle|^{2} \leq\left\langle N_{a}\right\rangle\left\langle N_{b}\right\rangle\left\langle N_{c}\right\rangle, \\
& |\langle a b\rangle\langle c\rangle|^{2} \leq\left\langle N_{a}\right\rangle\left\langle N_{b}\right\rangle\left\langle N_{c}\right\rangle, \\
& |\langle a c\rangle\langle b\rangle|^{2} \leq\left\langle N_{a}\right\rangle\left\langle N_{c}\right\rangle\left\langle N_{b}\right\rangle,
\end{aligned}
$$

then the pure state is fully entangled. 
Proof: Without loss of generality, we suppose that a $A \mid B C$ separable state satisfies the above three inequalities and the inequality (35), $|\langle a b c\rangle|>\left[\left\langle N_{a}\right\rangle\left\langle N_{b}\right\rangle\left\langle N_{c}\right\rangle\right]^{1 / 2}$ simultaneously. However for a $A \mid B C$ separable state, $|\langle a b c\rangle|=|\langle a\rangle\langle b c\rangle|$. This contradicts with (36), $|\langle a\rangle\langle b c\rangle|^{2} \leq\left\langle N_{a}\right\rangle\left\langle N_{b}\right\rangle\left\langle N_{c}\right\rangle$. This ends the proof.

We can extend these inequalities to general forms.

Corollary 10 If a state satisfies the following relations simultaneously,

$$
\begin{aligned}
& \left|\left\langle a^{m} b^{n} c^{l}\right\rangle\right|>\left[\left\langle\left(a^{\dagger}\right)^{m} a^{m}\right\rangle\left\langle\left(b^{\dagger}\right)^{n} b^{n}\right\rangle\left\langle\left(c^{\dagger}\right)^{l} c^{l}\right\rangle\right]^{1 / 2}, \\
& \left|\left\langle a^{m} b^{n}\right\rangle\left\langle c^{l}\right\rangle\right|^{2} \leq\left\langle\left(a^{\dagger}\right)^{m} a^{m}\right\rangle\left\langle\left(b^{\dagger}\right)^{n} b^{n}\right\rangle\left\langle\left(c^{\dagger}\right)^{l} c^{l}\right\rangle, \\
& \left|\left\langle a^{m}\right\rangle\left\langle b^{n} c^{l}\right\rangle\right|^{2} \leq\left\langle\left(a^{\dagger}\right)^{m} a^{m}\right\rangle\left\langle\left(b^{\dagger}\right)^{n} b^{n}\right\rangle\left\langle\left(c^{\dagger}\right)^{l} c^{l}\right\rangle, \\
& \left|\left\langle a^{m} c^{l}\right\rangle\left\langle b^{n}\right\rangle\right|^{2} \leq\left\langle\left(a^{\dagger}\right)^{m} a^{m}\right\rangle\left\langle\left(c^{\dagger}\right)^{l} c^{l}\right\rangle\left\langle\left(b^{\dagger}\right)^{n} b^{n}\right\rangle,
\end{aligned}
$$

where $m, n$ and $l$ are positive integers, we can conclude that the state is fully entangled.

Consider the state

$$
|\psi\rangle=N_{-}(\alpha, \beta, \gamma)(|\alpha, \beta, \gamma\rangle-|-\alpha,-\beta,-\gamma\rangle),
$$

where the normalization

$$
N_{-}=\left[2\left(1-e^{-2\left(|\alpha|^{2}+|\beta|^{2}+|\gamma|^{2}\right)}\right)\right]^{-1 / 2},
$$

$|\alpha, \beta, \gamma\rangle$ is the coherent state. For this state we have

$$
\begin{aligned}
\left|\left\langle a^{\dagger}(b)^{2} c\right\rangle\right|= & \mid \frac{1}{2\left(1-e^{-2\left(|\alpha|^{2}+|\beta|^{2}+|\gamma|^{2}\right)}\right)}\left(\alpha^{*}\langle\alpha, \beta, \gamma|+\alpha^{*}\langle-\alpha,-\beta,-\gamma|\right) \\
& \left(\beta^{2} \gamma|\alpha, \beta, \gamma\rangle+\beta^{2} \gamma|-\alpha,-\beta,-\gamma\rangle\right) \mid \\
= & \left|\frac{2 \alpha^{*} \beta^{2} \gamma\left(1+e^{-2\left(|\alpha|^{2}+|\beta|^{2}+|\gamma|^{2}\right)}\right)}{2\left(1-e^{-2\left(|\alpha|^{2}+|\beta|^{2}+|\gamma|^{2}\right)}\right)}\right|=\left|\alpha^{*} \beta^{2} \gamma\right| \operatorname{coth}\left(|\alpha|^{2}+|\beta|^{2}+|\gamma|^{2}\right)
\end{aligned}
$$

and

$$
\begin{aligned}
& \left(\left\langle a^{\dagger} a b^{\dagger} b^{\dagger} b b c^{\dagger} c\right\rangle\right)^{1 / 2} \\
= & \left(\frac{1}{2\left(1-e^{-2\left(|\alpha|^{2}+|\beta|^{2}+|\gamma|^{2}\right)}\right)}\left(\alpha^{*} \beta^{*} \beta^{*} \gamma^{*}\langle\alpha, \beta, \gamma|-\alpha^{*} \beta^{*} \beta^{*} \gamma^{*}\langle-\alpha,-\beta,-\gamma|\right)\right. \\
& \left.\left(\alpha \beta^{2} \gamma|\alpha, \beta, \gamma\rangle-\alpha \beta^{2} \gamma|-\alpha,-\beta,-\gamma\rangle\right)\right)^{1 / 2} \\
= & \left(\frac{2|\alpha|^{2}|\beta|^{4}|\gamma|^{2}\left(1-e^{-2\left(|\alpha|^{2}+|\beta|^{2}+|\gamma|^{2}\right)}\right)}{2\left(1-e^{-2\left(|\alpha|^{2}+|\beta|^{2}+|\gamma|^{2}\right)}\right)}\right)^{1 / 2}=|\alpha||\beta|^{2}|\gamma| .
\end{aligned}
$$


Eq. (46) is clearly less than Eq. (45) for all nonzero parameters. Hence, the state is $A \mid B C$ entangled from inequality (15). It is easily shown that for this state $\left|a^{2} b^{\dagger} c\right|^{2}>\left\langle a^{\dagger} a^{\dagger} a a N_{b} N_{c}\right\rangle$, $\left.\left|a^{2} b c^{\dagger}\right|^{2}\right\rangle\left\langle N_{a} N_{b} c^{\dagger} c^{\dagger} c c\right\rangle$. Therefore we conclude from (17) and (11) that this state is not only $A B \mid C$ entangled but also $B \mid A C$ entangled: this state is fully entangled.

\section{MULTI-MODE STATE}

The methods employed above for 3-mode states can be extended to n-mode states. Consider $n$ modes whose annihilation operators are $a_{1}, a_{2}, \cdots$ and $a_{n}$, respectively. For a state that is separable between $m$-mode and $(n-m)$-mode, we have that

$$
\begin{gathered}
\left|\left\langle a_{1} a_{2} \cdots a_{m} a_{m+1}^{\dagger} \cdots a_{n}^{\dagger}\right\rangle\right|^{2} \leq\left\langle N_{1} N_{2} \cdots N_{n}\right\rangle \\
\left|\left\langle a_{1} a_{2} \cdots a_{m} a_{m+1} \cdots a_{n}\right\rangle\right|^{2} \leq\left\langle N_{1} N_{2} \cdots N_{m}\right\rangle\left\langle N_{m+1} \cdots N_{n}\right\rangle .
\end{gathered}
$$

Therefore, if the inequalities (47) or (48) are violated, the state is entangled between $m$-mode and $(n-m)$-mode. We also have that for a general $m \mid(n-m)$ separable state

$$
\begin{gathered}
\left|\left\langle a_{1}^{l_{1}} a_{2}^{l_{2}} \cdots a_{m}^{l_{m}} \cdots\left(a_{n}^{\dagger}\right)^{l_{n}}\right\rangle\right|^{2} \leq\left\langle\left(a_{1}^{\dagger}\right)^{l_{1}} a_{1}^{l_{1}}\left(a_{2}^{\dagger}\right)^{l_{2}} a_{2}^{l_{2}} \cdots\left(a_{m}^{\dagger}\right)^{l_{m}} a_{m}^{l_{m}}\right\rangle, \\
\left|\left\langle a_{1}^{l_{1}} a_{2}^{l_{2}} \cdots a_{n}^{l_{n}}\right\rangle\right|^{2} \leq\left\langle\left(a_{1}^{\dagger}\right)^{l_{1}} a_{1}^{l_{1}}\left(a_{2}^{\dagger}\right)^{l_{2}} a_{2}^{l_{2}} \cdots\left(a_{m}^{\dagger}\right)^{l_{m}} a_{m}^{l_{m}}\right\rangle\left\langle\left(a_{m+1}^{\dagger}\right)^{l_{m+1}} a_{m+1}^{l_{m+1}} \cdots\left(a_{m}^{\dagger}\right)^{l_{m}} a_{m}^{l_{m}}\right\rangle .
\end{gathered}
$$

Denote $K(\phi)=e^{i \phi} a_{1} a_{2} \cdots a_{n}+e^{-i \phi} a_{1}^{\dagger} a_{2}^{\dagger} \cdots a_{n}^{\dagger}$, we have that for a fully separable state

$$
(\Delta K(\phi))^{2} \geq 1
$$

In analogy to formula (32), (51) holds for any separable states. Hence if the inequality (51) is violated, the multi-mode state must be fully entangled.

\section{CONCLUSIONS}

We have studied inseparability conditions for multi-mode states by presenting a series of inequalities. These conditions provide, in principle, measurable tests of entanglement, in the sense that all of the quantities appeared in the inequalities can be measured experimentally. The results in section 2 (theorems 4-7) have simple forms and can easily applied. They coincide with the ones in [10, 14, 15] that can be obtained by extending the methods used on non-positivity of partial transposition (NPT) in [14, 15]. In many practical cases, such 
as teleportation network, we firstly need to detect the fully entanglement of a state. Our theorem 8-9 could be applied for such purpose, as the quantities in the inequalities can be measured experimentally. For dealing with the entanglement of Gaussian states there are already very nice results $[4$, 5, 7, 8]. Our theorems 8-9 give some results which are derived for general states. Hence they could be applied for detecting entanglement of non-Gaussian states.

The work is partly supported by NKBRPC(2004CB318000) and NSFC projects $(10375038,90403018,10675086)$

[1] M. M. Wolf, G. Giedke,J. I. Cirac, Phys. Rev. Lett. 96, 080502(2006)

[2] G. Giedke, B. Kraus, M. Lewenstein and J. I. Cirac, Phys. Rev. Lett. 87, 167904(2001)

[3] P. Hyllus, and J. Eisert, New J. Phys. 8, 51(2006)

[4] R. Simon, Phys. Rev. Lett. 84, 2726(2000)

[5] L. M. Duan, G. Giedke, J. I. Cirac, P. Zoller, Phys. Rev. Lett. 84, 2722(2000)

[6] G. Giedke, B. Kraus, M. Lewenstein And J. I. Cirac Phys. Rev. A 64, 052303(2001)

[7] A. Ferraro, S. Olivares, M. G. A. Paris, Napoli Series on Physics and Astrophysics (Bibliopolis, Napoli, 2005), quant-ph/0503237

[8] A. Serafini, Phys. Rev. Lett. 96, 110402(2006)

[9] H. Yonezawa, T. Aoki, A. Furusawa, Nature(London)431,430(2004)

[10] P. van Loock and A. Furusawa, Phys. Rev. A 67, 052315(2003)

[11] H. F. Hofmann, K. Kojima, S. Takeuchi and K. Sasaki, Phys. Rev. A 68, 043813 (2003)

[12] O. Gühne, Phys. Rev. Lett. 92, 117903 (2004)

[13] M. Hillery and M. Zubairy, Phys. Rev. Lett. 96, 050503(2006)

[14] E. Shchukin and W. Vogel, Phys. Rev. Lett. 95, 230502(2006)

[15] E. Shchukin and W. Vogel, Phys. Rev. A 74, 030302(2006)

[16] A. Miranowicz, M. Piani, P. Horodecki, R. Horodecki, quant-ph/0605001

[17] S. L. Braunstein,P. van Loock, Rev. Mod. Phys.77, 513(2005)

[18] E. Shchukin and W. Vogel, Phys. Rev. A. 72, 043808(2005)

[19] J. Uffink, Phys. Rev. Lett. 88, 230406(2002)

[20] W. Dür, J. I. Cirac, R. Tarrach, Phys. Rev. Lett. 83, 3562(1999) 